\title{
Deterministic Enhancement of Coherent Photon Generation from a Nitrogen-Vacancy Center in Ultrapure Diamond
}

\author{
Daniel Riedel, ' Immo Söllner, Brendan J. Shields, Sebastian Starosielec, Patrick Appel, \\ Elke Neu, ${ }^{\dagger}$ Patrick Maletinsky, and Richard J. Warburton \\ Department of Physics, University of Basel, Klingelbergstrasse 82, Basel CH-4056, Switzerland
}

(Received 2 March 2017; published 7 September 2017)

\begin{abstract}
The nitrogen-vacancy (NV) center in diamond has an optically addressable, highly coherent spin. However, a NV center even in high-quality single-crystalline material is a very poor source of single photons: Extraction out of the high-index diamond is inefficient, the emission of coherent photons represents just a few percent of the total emission, and the decay time is large. In principle, all three problems can be addressed with a resonant microcavity. In practice, it has proved difficult to implement this concept: Photonic engineering hinges on nanofabrication, yet it is notoriously difficult to process diamond without degrading the NV centers. Here, we present a microcavity scheme that uses minimally processed diamond, thereby preserving the high quality of the starting material and a tunable microcavity platform. We demonstrate a clear change in the lifetime for multiple individual NV centers on tuning both the cavity frequency and antinode position, a Purcell effect. The overall Purcell factor $F_{P}=2.0$ translates to a Purcell factor for the zero phonon line (ZPL) of $F_{P}^{\mathrm{ZPL}} \sim 30$ and an increase in the ZPL emission probability from about $3 \%$ to $46 \%$. By making a step change in the NV's optical properties in a deterministic way, these results pave the way for much enhanced spin-photon and spin-spin entanglement rates.
\end{abstract}

DOI: 10.1103/PhysRevX.7.031040

The nitrogen-vacancy (NV) center in diamond constitutes a workhorse in quantum technology on account of its optically addressable, coherent electron spin [1]. The NV stands out for its long spin coherence times [2], robust single-photon emission [3] and the possibility of mapping its spin state to nearby nuclear spins [4]. Advances in spin-photon entanglement [5] and twophoton quantum interference protocols [6,7] pave the way for the implementation of quantum teleportation [8] and long-distance spin-spin entanglement [9]. However, the success rate of these protocols and the scaling up to extended networks are both limited by the very small generation rate of indistinguishable photons from individual NV centers [10].

There are at least four factors that limit the generation rate of indistinguishable photons. First, the lifetime of NV centers is relatively long, about 12 ns. Second, only a small fraction, about $3 \%-4 \%$, of the NV emission goes into the zero phonon line (ZPL) [11,12]. Only ZPL emission is useful for photon-based entanglement-swapping

\footnotetext{
*daniel.riedel@unibas.ch

${ }^{\dagger}$ Present address: Department of Physics, Saarland University, Campus E2 6, Saarbrücken DE-66123, Germany.

Published by the American Physical Society under the terms of the Creative Commons Attribution 4.0 International license. Further distribution of this work must maintain attribution to the author(s) and the published article's title, journal citation, and DOI.
}

Subject Areas: Condensed Matter Physics, Photonics, Quantum Information protocols as the phonon involved in non-ZPL emission dephases very rapidly. Third, the photon extraction efficiency out of the diamond is hindered by the large refractive index of diamond itself. Finally, there are random spectral fluctuations in the exact frequency of the NV emission caused by charge noise in the diamond host [6].

Coupling the NV center to a high-quality-factor, lowmode-volume optical microcavity offers a potential remedy to the first three factors, thereby dramatically improving the rate of coherent photon generation. These improvements depend on the weak-coupling regime of cavity quantum electrodynamics in which the emitter couples irreversibly to a single microcavity mode. The microcavity increases the total emission rate, the Purcell effect, and on resonance with the ZPL, the fraction of emission into the ZPL is likewise increased. The same coupling also enhances the ZPL extraction efficiency: Photons leaking out of the cavity are channeled into a single propagating mode. A notable feature is that, as compared to the strong-coupling regime of cavity quantum electrodynamics, modest cavity performance is all that is required $[13,14]$. Implementing these concepts for emitters in diamond has proved difficult so far. Nevertheless, coupling of NVs to photonic crystal cavities has been shown to convey significant improvements on account of the particularly small mode volume $V$ [15-19]. However, fabricating such structures exhibiting high quality factors ( $Q$-factors) in diamond is challenging: Diamond is a very hard and chemically inert material. Furthermore, 
achieving a spatial and spectral resonance with a single emitter is difficult, such that device yield is poor. Also, efficient outcoupling is hard to engineer. In addition, the invasive processing causes a worsening of the spectral fluctuations, particularly for NV centers, the fourth problem mentioned above.

In comparison, a miniaturized Fabry-Pérot microcavity has the advantage of in situ spatial and spectral tuning, along with high $Q$-factors and good mode matching to a propagating Gaussian beam, at the expense of an increased mode volume [20-22]. The feasibility of this approach has been demonstrated by enhancing the emission rate of emitters in nanocrystals [23-26]. However, as in photonic crystal cavities, NVs in nanocrystals typically suffer from significant line broadening owing to their close proximity to fluctuating charges at the surface. These spectral fluctuations are so severe that schemes involving photon-based entanglement swapping have only been successfully implemented using high-purity single-crystalline diamond material $[27,28]$.

Here, we present deterministic enhancement of the ZPL emission rate from single NV centers with narrow ZPL line widths (about $1 \mathrm{GHz}$ ) by resonant coupling to a high- $Q$ microcavity mode. We demonstrate an increase of the probability of ZPL emission to about $46 \%$. Two principles have guided our work. First, at this stage of diamond-based quantum technology, in situ tuning of both microcavity frequency and antinode position is extremely valuable. We have therefore opted for a miniaturized Fabry-Pérot microcavity [Fig. 1(a)]. Second, we use ultrapure diamond material with minimal processing in order to ensure good NV optical properties. Specifically, thin diamond membranes are created out of high-purity, single-crystalline chemical vapor deposition (CVD) diamond. As starting material, we employ commercially available CVD diamond [Element 6, (100)-orientation] and introduce NV centers at a target depth of $68 \mathrm{~nm}$ by nitrogen implantation $\left({ }^{14} \mathrm{~N}\right.$, $55 \mathrm{keV}, 2 \times 10^{9}$ ions $/ \mathrm{cm}^{2}$, straggle $16 \mathrm{~nm}$ ). Using multistep high-temperature annealing, NV centers with close-tolifetime-limited emission line widths can be created [29]. Here, photoluminescence excitation (PLE) scans of NVs in the starting material yield long-term ZPL line widths of $\lesssim 100 \mathrm{MHz}$ at $4 \mathrm{~K}$ [Fig. 1(b)]. Membranes of thickness $t_{\mathrm{d}} \lesssim 1 \mu \mathrm{m}$ (with typical lateral dimensions $20 \times 20 \mu \mathrm{m}^{2}$ ) and surface roughness of $\lesssim 0.3 \mathrm{~nm}$ are fabricated from this starting material by plasma etching and microstructuring [30-32]. Using a micromanipulator, we break out membranes [Fig. 1(c)] and transfer them to a planar mirror to which they adhere by van der Waals forces [32] [Fig. 1(d)]. Individual NV centers in the membranes have PLE line widths of about $1 \mathrm{GHz}$ [Fig. 1(b)], higher than the line widths in the starting material but still much lower than typical line widths in diamond nanocrystals. Notably, these line widths are smaller than the ground-state spintriplet splitting of $2.87 \mathrm{GHz}$, an essential feature for quantum information applications [17]. We note that in our

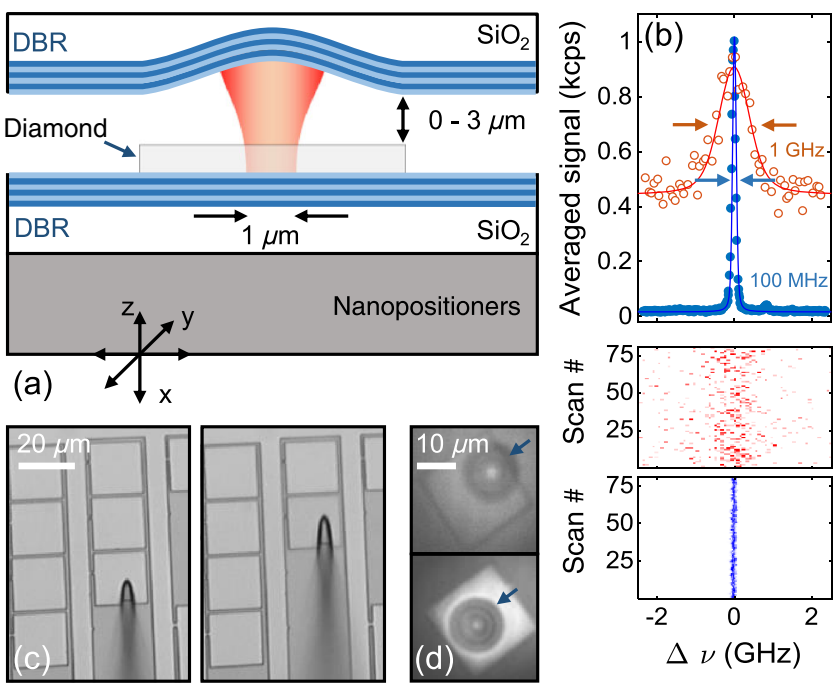

FIG. 1. (a) Schematic of the tunable microcavity containing a thin diamond membrane. Both the antinode location and resonance frequency of the microcavity mode can be tuned in situ. (b) PLE scans of near-surface $(68 \mathrm{~nm}) \mathrm{NV}$ centers in unprocessed diamond (blue symbols; the lower panel: resonant laser power $3 \mathrm{nW}$ and scan speed $340 \mathrm{MHz} / \mathrm{s}$ ) and in microstructured $\left(t_{\mathrm{d}} \leq 1 \mu \mathrm{m}\right)$ diamond (red symbols; the central panel: resonant laser power $10 \mathrm{nW}$ and scan speed $7.8 \mathrm{GHz} / \mathrm{s}$ ), yielding averaged zero-phonon line widths of about $100 \mathrm{MHz}$ and $1 \mathrm{GHz}$, respectively (upper panel). (c) Detaching a $20 \times 20 \mu \mathrm{m}^{2}$ membrane using a micromanipulator. (d) Images (recorded with a wavelength outside the DBR stop band) of the diamond microcavity demonstrating the in situ control of the lateral position. The arrows indicate the position of the concave top mirror.

PLE pulse sequence, we apply a green repump pulse for every step of the resonant laser. This randomizes the charge environment for every pixel in the line scan. Thus, we capture all spectral shifts arising from the charge fluctuations. We anticipate that a significant reduction can be achieved by applying resonant repump techniques [33] and by electronic feedback stabilization via Stark effect tuning [34].

The miniaturized Fabry-Pérot cavity consists of a plane bottom mirror and a concave top mirror with radius of curvature $R=16 \mu \mathrm{m}$ [Fig. 1(a)]. The curved top mirror is fabricated by creating a concave depression in a silica substrate with laser ablation followed by mirror coating $[20,21,35]$. Both bottom and top mirrors are distributed Bragg reflectors (DBRs) with reflectivity $>99.99 \%$. The bare cavity has a finesse of $F \gtrsim 10000$. The microcavity can be tuned in situ with a set of three-axis nanopositioners [Fig. 1(a)]. Additionally, the entire microcavity can be moved in situ with respect to a fixed objective lens, which allows for optimizing mode matching between the external excitation or detection mode and the microcavity mode [20-22]. The compact cavity design facilitates lowtemperature experiments in a liquid-helium bath cryostat. 

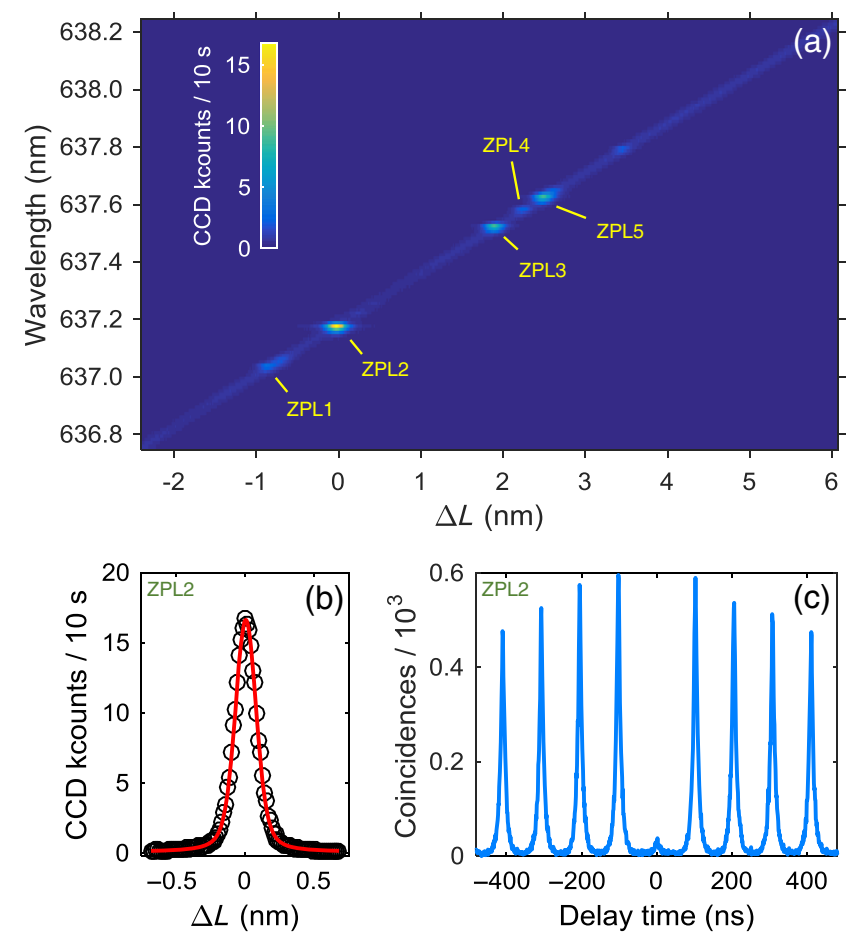

FIG. 2. (a) PL spectra around the ZPL transition for different air-gap detunings $\Delta L$ about $L=1.96 \mu \mathrm{m}$. Each resonance corresponds to the ZPL emission of a single NV center, as labeled. The PL is excited using a pulsed supercontinuum laser source $(P \sim 10 \mathrm{~mW}, \lambda=560 \pm 20 \mathrm{~nm})$ and detected by a grating spectrometer. (b) Integrated PL versus $L$ for ZPL2 along with a Voigt fit (FWHM Lorentzian contribution $\Gamma_{L}=60.6 \mathrm{pm}$ ). Together with the cavity dispersion in (a), $\Gamma_{L}$ determines the cavity $Q$-factor, $Q=\lambda / \Gamma_{\lambda}=\lambda /\left(\Gamma_{L} \cdot \mathrm{d} \lambda / \mathrm{d} L\right)=58500$. (c) Photon autocorrelation $\left[g^{(2)}(t)\right]$ measurement on ZPL2 exhibiting a clear single-photon emission $g^{(2)}(0)=0.27$. The ZPL emission is filtered $(637 \pm 7 \mathrm{~nm})$ and analyzed via a Hanbury BrownTwiss setup (integration time: $45000 \mathrm{~s}$ ).

Figure 2(a) shows photoluminescence (PL) from the diamond membrane microcavity while tuning the width of the air gap $L$. The spectra are recorded for the lowest attainable fundamental microcavity mode that comes into resonance with the different ZPL transitions. Notably here, the two orthogonal cavity polarizations are degenerate, which allows for full control over the light polarization. The mirrors are almost in physical contact such that $L$ is dominated by the depth of the curved top mirror (about $1 \mu \mathrm{m})$. Spectra were recorded on tuning the microcavity by changing the membrane-top mirror separation, and hence $L$. Weak PL is observed at all $L$ and arises from broadband emission from the diamond membrane. It allows the $L$ dependence of the microcavity mode to be characterized [Fig. 2(a)].

In addition to the weak broadband emission displayed in Fig. 2(a), there are sharp features at specific $L$ which we assign to individual ZPL transitions. The PL from ZPL2 at $L=1.96 \mu \mathrm{m}$ is shown for different air-gap detunings $\Delta L$ in Fig. 2(b). We fit a Voigt profile to the resonance (the Gaussian component accounts for the low-frequency acoustic noise). The full width at half maximum (FWHM) of the Lorentzian contribution is $\Gamma_{L}=60.6 \mathrm{pm}$, yielding a finesse of $F=5260$. We determine the $Q$-factor of the cavity according to $Q=\lambda / \Gamma_{\lambda}=\lambda /\left(\Gamma_{L} \cdot \mathrm{d} \lambda / \mathrm{d} L\right)=2 F /(\mathrm{d} \lambda / \mathrm{d} L)=$ 58500 , with $\mathrm{d} \lambda / \mathrm{d} L=0.18$. For fixed $L$, for instance at ZPL2, the microcavity line width is $\Gamma_{f}=8.0 \mathrm{GHz}$.

We confirm that the observed resonances are associated with single quantum emitters by performing a photon autocorrelation $\left[g^{(2)}(t)\right]$ measurement with a Hanbury Brown-Twiss setup. The results on ZPL2 are shown in Fig. 2(c). The strong suppression of the peak at zero delay is a clear signature of single-photon emission. The data are analyzed quantitatively by calculating the pulse area of each peak and normalizing the data to the averaged peak area at long delay times $(500 \mu \mathrm{s})$. This gives $g^{(2)}(0)=$ 0.24 , comfortably less than 0.5 . Away from $t=0, g^{(2)}(t)$ is initially larger than one with $g^{(2)}(103 \mathrm{~ns})=3.93$, which is a bunching behavior. This bunching decays as $t$ increases, signifying telegraph noise between bright and dark states. The dark states are the singlet shelving states and the neutral charge state [3]. In the bright state, the important quantity is $g$, the ratio of the probability of generating two photons in the same pulse to the probability of generating one photon in each of two successive pulses. We find here a small value, $g=0.06$.

The spectral analysis shows that the microcavity acts as a narrow spectral filter. It is crucial, however, to demonstrate that the microcavity modifies the behavior of the emitter itself. The key parameter in the weak-coupling regime is the decay rate. We therefore turn to excited-state lifetime measurements of individual NV centers and tune both the resonance frequency (via an in situ change of $L$ ) and the lateral position of the cavity antinode [via an in situ change in $(x, y)$ ]. Figure 3(a) shows decay curves for ZPL2 for several different detunings $\Delta L$. There is a clear change in lifetime. To extract the lifetime quantitatively, we note that for large detunings $\Delta L$, the weak background emission represents a significant part of the signal. However, the background emission decays very rapidly: The slower decay process arises from the decay of the NV center. The data for delays larger than $3 \mathrm{~ns}$ are fitted to a single exponential convoluted with the instrumental response [36]. The lifetime decreases on detuning $\Delta L$ from $10.4 \mathrm{~ns}$ to $7.06 \mathrm{~ns}$. This is clear evidence of a Purcell effect.

This assertion is backed up by recording the decay time over a larger range of $\Delta L$ [Fig. 3(b)]. Five ZPLs come into resonance with the microcavity at different values of $\Delta L$, and each shows a clear Purcell effect, an enhanced decay rate $\gamma_{R}$ when in spectral resonance with the microcavity. Each resonance is well described with a Lorentzian function of $\Delta L$. Figure 3(c) shows the results of the alternative experiment, detuning in lateral position $\Delta x$ while maintaining the spectral resonance $(\Delta L=0)$, on 

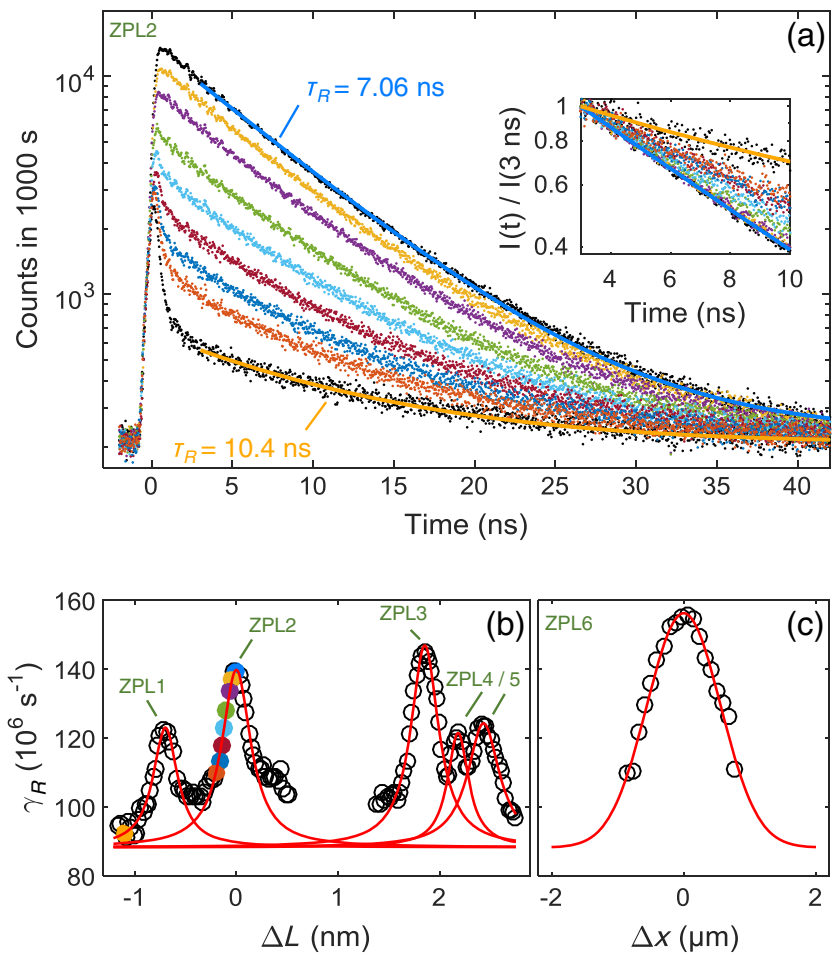

FIG. 3. (a) PL decay curves of ZPL2 following pulsed excitation as a function of cavity-length detuning $\Delta L$ for an acquisition time of $1000 \mathrm{~s}$. The data for delays larger than $3 \mathrm{~ns}$ are fitted to a single-exponential convoluted with the instrumental response. The inset shows the normalized decay curves highlighting the clear change of the decay rate on changing $\Delta L$. (b) Recombination rate $\gamma_{\mathrm{R}}$ versus $\Delta L$ for a fixed lateral position. For each ZPL resonance, $\gamma_{R}$ exhibits a Purcell effect. The experimental data are fitted to Lorentzian curves with FWHM $\Gamma_{L}=(0.32 \pm 0.05) \mathrm{nm}$. The color of the symbols matches the decay curves in panel (a). (c) Recombination rate versus lateral position detuning $\Delta x$ on ZPL6 for zero spectral detuning. The experimental data are fitted to a Gaussian with FWHM $0.80 \mu \mathrm{m}$.

ZPL6, a NV located at a different location in the diamond membrane. These results also show a Purcell effect, a resonance in $\gamma_{R}$ as a function of $\Delta x$. The $\gamma_{R}$ versus $\Delta x$ data are well fitted by a Gaussian function with FWHM $\Gamma_{x}=0.80 \mu \mathrm{m}$, which represents the lateral extent of the mode in the microcavity. ZPL6 exhibits the largest decay rate on resonance, $\gamma_{R}^{\text {on }}=158 \times 10^{6} \mathrm{~s}^{-1}$ : This suggests that this $\mathrm{NV}$ is positioned close to the optimal depth in the diamond membrane.

We interpret the results in terms of a Purcell enhancement factor $F_{P}$. Without the top mirror, the decay time is consistently $\tau_{R}^{0}=12.6 \mathrm{~ns}$ for all NVs corresponding to a decay rate $\gamma_{R}^{0}=79.4 \times 10^{6} \mathrm{~s}^{-1}$. In terms of the total decay rates, $F_{P}=\gamma_{R}^{\text {on }} / \gamma_{R}^{0}=2.0$. We note that, in the microcavity, the decay rate in the limit of large $\Delta L$ detunings is slightly larger than the bulk decay rate [Fig. 3(b)]. We attribute this to non-ZPL emission, which is Purcell enhanced not just at the fundamental microcavity mode but also at higher modes.
This modest Purcell enhancement of the total decay rate, $F_{P}=2.0$, masks the large changes to the ZPL emission rate. Without the microcavity, radiative decay occurs predominantly into laterally propagating modes, modes defined by the membrane itself. With the microcavity, these lateral modes still exist of course, and the decay rate into the lateral modes is unchanged. Instead, the microcavity boosts the radiative decay rate into a single, vertically propagating mode. Even a small Purcell factor implies that the decay rate into the single microcavity mode dominates over the decay rate into all the lateral modes. With the microcavity tuned to the ZPL, this implies a large change to the ZPL fraction.

We analyze the data to determine the ZPL Purcell factor and the ZPL fraction when the microcavity is tuned into resonance with the ZPL. When the microcavity is tuned into resonance with the ZPL line, the "on" state, the total decay rate is $\gamma_{R}^{\text {on }}=\gamma_{0}^{\text {on }}+\gamma_{1}^{\text {on }}$, where $\gamma_{0}^{\text {on }}$ is the ZPL emission rate and $\gamma_{1}^{\text {on }}$ the non-ZPL decay rate. When the microcavity is tuned far out of resonance with the ZPL line, the "off" state, the decay rate is $\gamma_{R}^{\text {off }}=\gamma_{0}^{\text {off }}+\gamma_{1}^{\text {off }}$. The experiment determines $\gamma_{R}^{\text {on }}$ and, by fitting $\gamma_{R}$ as a function of either $\Delta L$ or $\Delta x$ (Fig. 3), $\gamma_{R}^{\text {off }}=88.2 \times 10^{6} \mathrm{~s}^{-1}$. To proceed, we note, first, that the non-ZPL emission is so broadband that the small frequency shift of the microcavity mode between the "on" and "off" states makes no change to the non-ZPL decay rate, i.e., $\gamma_{1}^{\text {on }}=\gamma_{1}^{\text {off }}$. Second, in the "off" state, ZPL emission takes place predominantly into laterally propagating modes. This is also the case in a bare membrane such that $\gamma_{0}^{\text {off }} \approx \gamma_{0}$, where $\gamma_{0}$ is the ZPL emission rate in the bare membrane. This allows us to determine the Purcell factor for the ZPL alone: $F_{P}^{\mathrm{ZPL}}=\gamma_{0}^{\mathrm{on}} / \gamma_{0}=\left(\gamma_{R}^{\mathrm{on}}-\gamma_{R}^{\text {off }}\right) / \gamma_{0}+1$. Taking $\gamma_{0}$ as $2.4 \%(5 \%)$ of $\gamma_{R}^{0}$, the range of reported NV Debye-Waller factors [36] leads to $\gamma_{0}=1.91 \times 10^{6} \mathrm{~s}^{-1}\left(3.97 \times 10^{6} \mathrm{~s}^{-1}\right)$ and hence $F_{P}^{\mathrm{ZPL}}=37.7$ (18.6) (using the experimentally determined values for ZPL6: $\gamma_{R}^{\text {off }}=88.2 \times 10^{6} \mathrm{~s}^{-1}$, $\gamma_{R}^{\text {on }}=158 \times 10^{6} \mathrm{~s}^{-1}$ ). The fraction of photons emitted into the ZPL in the "on" state is $\eta_{\mathrm{ZPL}}=F_{P}^{\mathrm{ZPL}} \times \gamma_{0} / \gamma_{R}^{\mathrm{on}}$. For ZPL6, $\eta_{\mathrm{ZPL}}=45.4 \%$ (46.7\%). We note that $\eta_{\mathrm{ZPL}}$, a more important parameter for quantum photonics than $F_{P}^{Z P L}$, depends very weakly on $\gamma_{0}$, which is not known precisely.

We attempt to account quantitatively for the experimental value of $F_{P}^{\mathrm{ZPL}}$. The key parameter is the vacuum electric field in the microcavity at the location of the NV center. The vacuum field is a sensitive function of the microcavity geometry, which in turn determines the mode structure. In particular, the separation of the fundamental microcavity modes depends on the width of the air gap, and the frequency separation between the fundamental modes and the higherorder modes depends on the radius of curvature of the top mirror. In addition, the mode structure on making large changes to $L$ (which can be described as an anticrossing between air-confined and diamond-confined modes [37]) depends sensitively on the diamond membrane thickness.

To measure the mode structure, we excite the diamond microcavity with a high power of green $(560 \mathrm{~nm})$ light and 


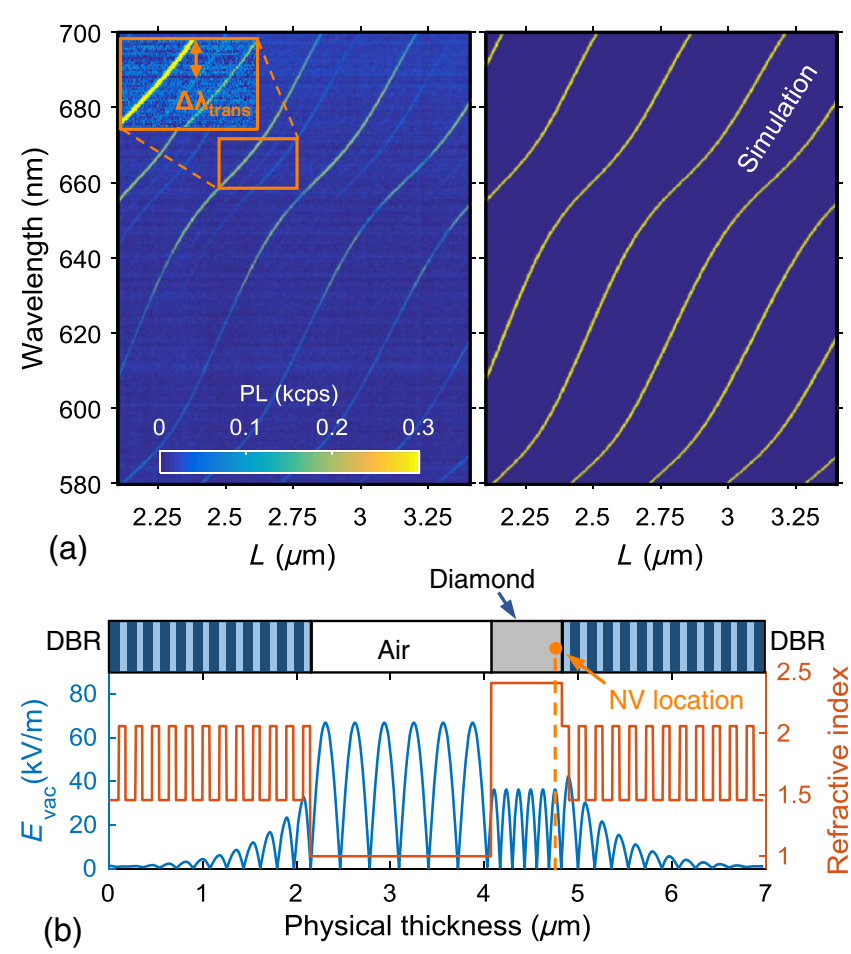

FIG. 4. (a) Left panel: Measured PL spectra on tuning the microcavity length $L$ over a wide range. The inset highlights the higher-order lateral modes. Right panel: Calculated mode dispersion. (b) The layer structure of the microcavity along with the refractive index dependence on $z$. The vacuum electric field $E_{\mathrm{vac}}$ is plotted against $z$ for $(x, y)=(0,0)$ for the lowest attainable fundamental microcavity mode. Parameters: Diamond thickness $t_{d}=0.77 \mu \mathrm{m}$, air-gap thickness $L=1.96 \mu \mathrm{m}$, diamond refractive index $n_{d}=2.41$, refractive indices of Bragg mirror $\left(n_{H}, n_{L}\right)=(2.06,1.46)$, and radius of curvature of top mirror $R=16 \mu \mathrm{m}$.

use the weak broadband emission from the diamond as an internal light source. Figure 4(a) shows the microcavity resonances as a function of wavelength and cavity length spanning several free spectral ranges. The fundamental microcavity modes along with the associated higher-order modes are clearly observed [Fig. 4(a)]. We calculate the mode structure by describing the longitudinal standing waves with a transfer-matrix calculation and the longitudinal confinement with Gaussian optics. We achieve excellent agreement with the experiment with $R=$ $16 \mu \mathrm{m}$ (matching the physical curvature of the mirror), air-gap thickness $L=1.96 \mu \mathrm{m}$ and $t_{d}=0.77 \mu \mathrm{m}$ (corresponding closely to the physical thickness determined with a scanning confocal microscope), and a FWHM beam waist of $0.83 \mu \mathrm{m}$ (corresponding to the value determined in situ on ZPL6) [Fig. 4(b)]. This leads to a maximum vacuum field in the diamond of $E_{\mathrm{vac}}=36.2 \mathrm{kV} / \mathrm{m}$.

The measurement of $\gamma_{R}^{0}$ enables the optical dipole moment of the NV center to be determined, $d_{\mathrm{NV}} / e=0.108 \mathrm{~nm}$. The optical dipoles of the NV centers are randomly oriented in a plane orthogonal to the $\mathrm{NV}$ axis
[32]. For NV centers that are coupled maximally to the cavity mode, the optical dipole of the ZPL is parallel to the diamond surface. We determine a coupling rate of the ZPL to the vacuum field of $g=d_{\mathrm{NV}} E_{\mathrm{vac}}=5.97 \times 10^{9} \mathrm{~s}^{-1}$. The vacuum field corresponds to an effective mode volume of $\hbar \omega /\left(2 \epsilon_{0} n^{2} E_{\mathrm{vac}}^{2}\right)=125(\lambda / n)^{3}[14]$. Here, we assume a unity internal quantum efficiency of the NV in accordance with a recent extrapolation of measured quantum efficiencies for shallow NVs into the bulk [38]. The measured $Q$-factor determines the photon decay rate out of the microcavity, $\kappa=2 \pi \Gamma_{\mathrm{f}}=5.06 \times 10^{10} \mathrm{~s}^{-1}$. In the case that emission into "leaky" lateral modes is unchanged by the microcavity, the Purcell factor [39] is $F_{P}^{\mathrm{ZPL}}=4 g^{2} /\left(\kappa \gamma_{R}^{0}\right)+1=36.5$, close to the value determined in the experiment. We note, however, that $F_{P}^{\mathrm{ZPL}}$ is associated with a systematic error due to the uncertainty of the Debye-Waller factor, the fraction of photons emitted into the ZPL. In fact, the argument can be turned around. The properties of the microcavity are so well understood [Figs. 4(a) and 4(b)] that a measurement of $F_{P}^{\mathrm{ZPL}}$ constitutes a measurement of $\gamma_{0} / \gamma_{R}^{0}$, the Debye-Waller factor. We find $\gamma_{0} / \gamma_{R}^{0}=2.55 \%$, which lies within the range of previous estimates [36].

The ultimate goal is to increase $\eta_{\mathrm{ZPL}}$ towards $100 \%$ and to collect as many of the ZPL photons as possible. In the present experiment, $\eta_{\text {ZPL }}$ is increased to about $46 \%$. The photon flux is limited by losses (by absorption or scattering) in the dielectric mirror. This loss can be eliminated with better mirrors: $10 \mathrm{ppm}$ loss dielectric mirrors are available. The present design [Fig. 4(b)] results in a node in the vacuum electric field at the diamond-vacuum interface in order to minimize the scattering losses. The calculations predict that by decreasing the diamond membrane thickness $t_{d}$ and employing state-of-the-art laser ablation techniques for fabricating shallow depressions with a smaller radius of curvature (depth of about $400 \mathrm{~nm}, R \sim 5.5 \mu \mathrm{m}$ [40]), the maximum vacuum field $E_{\mathrm{vac}}$ in the diamond is significantly boost. For instance, decreasing $t_{d}$ from $0.77 \mu \mathrm{m}$ to $3 \lambda_{\mathrm{ZPL}} / 4=198 \mathrm{~nm}$ in combination with the aforementioned improved microcavity design, allowing for $L=3 \lambda_{\mathrm{ZPL}} / 4=$ $478 \mathrm{~nm}$, increases the maximum vacuum field from $E_{\mathrm{vac}}=$ $36.2 \mathrm{kV} / \mathrm{m}$ to $E_{\mathrm{vac}}=85.7 \mathrm{kV} / \mathrm{m}$. We estimate that photon collection is maximized for $\kappa=2 g$, which requires a $Q$-factor of only 128000 ; the resulting Purcell factor and ZPL emission probability are $F_{P}^{\mathrm{ZPL}}=356$ and $\eta_{\mathrm{ZPL}}=87.9 \%$, respectively. If the diamond surface can be made sufficiently smooth, then it becomes possible to change the design such that there is an antinode at the diamond-vacuum interface. This boosts the vacuum electric field at the antinode in the diamond membrane: The global maximum of the vacuum field is now located in the diamond rather than in the air gap [20]. For instance, for $t_{d}=\lambda_{\mathrm{ZPL}} / 2=132 \mathrm{~nm}, L=\lambda_{\mathrm{ZPL}}=$ $637 \mathrm{~nm}, E_{\mathrm{vac}}=127 \mathrm{kV} / \mathrm{m}$ and, again choosing $\kappa=2 g$ (requiring $Q=86500$ ), $F_{P}^{\mathrm{ZPL}}=527$, and $\eta_{\mathrm{ZPL}}=91.5 \%$. In addition to providing a massive boost to the ZPL fraction, a further advantage of a large Purcell enhancement is that the 
$\mathrm{NV}$ transform limit increases, for $F_{P}^{\mathrm{ZPL}}=527(356)$ to $\Gamma_{f}=182 \mathrm{MHz}(127 \mathrm{MHz})$. Implementing these improvements would therefore mitigate the constraints on the spectral stability of the NV ZPL transition. Only a slight improvement on the line widths reported here is required in order to create a high fraction of indistinguishable photons. In this case, the demonstrated increase in the ZPL fraction by a factor of 15 in combination with an estimated threefold enhancement in the collection efficiency would boost the spin-spin entanglement rate by a factor of approximately 2000 compared to previous experiments [41].

Based on the work presented here, we propose that a miniaturized Fabry-Pérot microcavity using a thin diamond membrane constitutes a key building block for quantum technology applications. It has been proposed recently that the creation of the first metropolitan-scale quantum internet consisting of several nodes based on NV centers is within reach [42]. In the longer term, a quantum internet built using this approach depends on entanglement distillation [43], high-efficiency photon frequency conversion to the infrared [44], and coupling NV centers to a photonic cavity. The last point is demonstrated here. Cavity coupling confers general benefits to solid-state emitters. We point out that the miniaturized Fabry-Pérot microcavity has a rather generic design such that cavity enhancement is also an immediate possibility with other color centers in, for instance, diamond [45-47] and $\mathrm{SiC}$ [48-50].

\section{ACKNOWLEDGMENTS}

We acknowledge financial support from NCCR QSIT, a competence center funded by SNF, the Swiss Nanoscience Institute (SNI), the EU FP7 project DIADEMS (Grant No. 611143), ITN networks S3NANO and SpinNANO, and SNF Grants No. 200021_143697 and No. 200021_169321.

[1] F. Jelezko, T. Gaebel, I. Popa, A. Gruber, and J. Wrachtrup, Observation of Coherent Oscillations in a Single Electron Spin, Phys. Rev. Lett. 92, 076401 (2004).

[2] G. Balasubramanian, P. Neumann, D. Twitchen, M. Markham, R. Kolesov, N. Mizuochi, J. Isoya, J. Achard, J. Beck, J. Tissler et al., Ultralong Spin Coherence Time in Isotopically Engineered Diamond, Nat. Mater. 8, 383 (2009).

[3] C. Kurtsiefer, S. Mayer, P. Zarda, and H. Weinfurter, Stable Solid-State Source of Single Photons, Phys. Rev. Lett. 85, 290 (2000).

[4] M. V. G. Dutt, L. Childress, L. Jiang, E. Togan, J. Maze, F. Jelezko, A. S. Zibrov, P. R. Hemmer, and M. D. Lukin, Quantum Register Based on Individual Electronic and Nuclear Spin Qubits in Diamond, Science 316, 1312 (2007).

[5] E. Togan, Y. Chu, A. S. Trifonov, L. Jiang, J. Maze, L. Childress, M. V. G. Dutt, A. S. Sørensen, P. R. Hemmer, A.S. Zibrov, and M.D. Lukin, Quantum Entanglement Between an Optical Photon and a Solid-State Spin Qubit, Nature (London) 466, 730 (2010).
[6] H. Bernien, L. Childress, L. Robledo, M. Markham, D. Twitchen, and R. Hanson, Two-Photon Quantum Interference from Separate Nitrogen Vacancy Centers in Diamond, Phys. Rev. Lett. 108, 043604 (2012).

[7] A. Sipahigil, M. L. Goldman, E. Togan, Y. Chu, M. Markham, D. J. Twitchen, A. S. Zibrov, A. Kubanek, and M. D. Lukin, Quantum Interference of Single Photons from Remote Nitrogen-Vacancy Centers in Diamond, Phys. Rev. Lett. 108, 143601 (2012).

[8] W. Pfaff, B. Hensen, H. Bernien, S. B. van Dam, M. S. Blok, T. H. Taminiau, M. J. Tiggelman, R. N. Schouten, M. Markham, D. J. Twitchen, and R. Hanson, Unconditional Quantum Teleportation Between Distant Solid-State Qubits, Science 345, 532 (2014).

[9] B. Hensen, H. Bernien, A. E. Dréau, A. Reiserer, N. Kalb, M. S. Blok, J. Ruitenberg, R. F. L. Vermeulen, R. N. Schouten, C. Abellán et al., Experimental Loophole-Free Violation of a Bell Inequality Using Entangled Electron Spins Separated by $1.3 \mathrm{~km}$, Nature (London) 526, 682 (2015).

[10] W. B. Gao, A. Imamoglu, H. Bernien, and R. Hanson, Coherent Manipulation, Measurement and Entanglement of Individual Solid-State Spins Using Optical Fields, Nat. Photonics 9, 363 (2015).

[11] P. E. Barclay, K.-M. C. Fu, C. Santori, A. Faraon, and R. G. Beausoleil, Hybrid Nanocavity Resonant Enhancement of Color Center Emission in Diamond, Phys. Rev. X 1, 011007 (2011).

[12] H. Kaupp, C. Deutsch, H. C. Chang, J. Reichel, T. W. Hänsch, and D. Hunger, Scaling Laws of the Cavity Enhancement for Nitrogen-Vacancy Centers in Diamond, Phys. Rev. A 88, 053812 (2013).

[13] C.-H. Su, A. D. Greentree, and L. C. L. Hollenberg, Towards a Picosecond Transform-Limited Nitrogen-Vacancy Based Single Photon Source, Opt. Express 16, 6240 (2008).

[14] G. Khitrova, H. M. Gibbs, M. Kira, S. W. Koch, and A. Scherer, Vacuum Rabi Splitting in Semiconductors, Nat. Phys. 2, 81 (2006).

[15] A. Sipahigil, R. E. Evans, D. D. Sukachev, M. J. Burek, J. Borregaard, M. K. Bhaskar, C. T. Nguyen, J. L. Pacheco, H. A. Atikian, C. Meuwly et al., Single-Photon Switching and Entanglement of Solid-State Qubits in an Integrated Nanophotonic System, Science 354, 847 (2016).

[16] J. Riedrich-Möller, S. Pezzagna, J. Meijer, C. Pauly, F. Mücklich, M. Markham, A. M. Edmonds, and C. Becher, Nanoimplantation and Purcell Enhancement of Single NV Centers in Photonic Crystal Cavities in Diamond, Appl. Phys. Lett. 106, 221103 (2015).

[17] A. Faraon, C. Santori, Z. Huang, V. M. Acosta, and R. G. Beausoleil, Coupling of Nitrogen-Vacancy Centers to Photonic Crystal Cavities in Monocrystalline Diamond, Phys. Rev. Lett.109, 033604 (2012).

[18] B. J. M. Hausmann, B. J. Shields, Q. Quan, Y. Chu, N. P. De Leon, R. Evans, M. J. Burek, A. S. Zibrov, M. Markham, D. J. Twitchen et al., Coupling of NV Centers to Photonic Crystal Nanobeams in Diamond, Nano Lett. 13, 5791 (2013).

[19] L. Li, T. Schröder, E. H. Chen, M. Walsh, I. Bayn, J. Goldstein, O. Gaathon, M. E. Trusheim, M. Lu, J. Mower, 
M. Cotlet et al., Coherent Spin Control of a NanocavityEnhanced Qubit in Diamond, Nat. Commun. 6, 6173 (2014).

[20] R. J. Barbour, P. A. Dalgarno, A. Curran, K. M. Nowak, H. J. Baker, D. R. Hall, N. G. Stoltz, P. M. Petroff, and R. J. Warburton, A tunable microcavity, J. Appl. Phys. 110, 053107 (2011).

[21] L. Greuter, S. Starosielec, D. Najer, A. Ludwig, L. Duempelmann, D. Rohner, and R. J. Warburton, A Small Mode Volume Tunable Microcavity: Development and Characterization, Appl. Phys. Lett. 105, 121105 (2014).

[22] L. Greuter, S. Starosielec, A. V. Kuhlmann, and R. J. Warburton, Towards High Cooperativity Strong Coupling of a Quantum Dot in a Tunable Microcavity, Phys. Rev. B 92, 045302 (2015).

[23] R. Albrecht, A. Bommer, C. Deutsch, J. Reichel, and C. Becher, Coupling of a Single Nitrogen-Vacancy Center in Diamond to a Fiber-Based Microcavity, Phys. Rev. Lett. 110, 243602 (2013).

[24] S. Johnson, P. R. Dolan, T. Grange, A. A. P. Trichet, G. Hornecker, Y. C. Chen, L. Weng, G. M. Hughes, A. A. R. Watt, A. Auffèves, and J. M. Smith, Tunable Cavity Coupling of the Zero Phonon Line of a Nitrogen-Vacancy Defect in Diamond, New J. Phys. 17, 122003 (2015).

[25] H. Kaupp, T. Hümmer, M. Mader, B. Schlederer, J. Benedikter, P. Haeusser, H. C. Chang, H. Fedder, T. W. Hänsch, and D. Hunger, Purcell-Enhanced Single-Photon Emission from Nitrogen-Vacancy Centers Coupled to a Tunable Microcavity, Phys. Rev. Applied 6, 054010 (2016).

[26] J. Benedikter, H. Kaupp, T. Hümmer, Y. Liang, A. Bommer, C. Becher, A. Krueger, J. M. Smith, T. W. Hänsch, and D. Hunger, Cavity-Enhanced Single-Photon Source Based on the Silicon-Vacancy Center in Diamond, Phys. Rev. Applied 7, 024031 (2017).

[27] A. D. Greentree, P. Olivero, M. Draganski, E. Trajkov, J. R. Rabeau, P. Reichart, B. C. Gibson, S. Rubanov, S. T. Huntington, D. N. Jamieson, and S. Prawer, Critical Components for Diamond-Based Quantum Coherent Devices, J. Phys. Condens. Matter 18, S825 (2006).

[28] T. Schröder, S. Mouradian, J. Zheng, M. E. Trusheim, M. Walsh, E. H. Chen, L. Li, I. Bayn, and D. Englund, Review Article: Quantum Nanophotonics in Diamond, J. Opt. Soc. Am. B 33, B65 (2016).

[29] Y. Chu, N. P. De Leon, B. J. Shields, B. Hausmann, R. Evans, E. Togan, M. J. Burek, M. Markham, A. Stacey, A. S. Zibrov et al., Coherent Optical Transitions in Implanted Nitrogen Vacancy Centers, Nano Lett. 14, 1982 (2014).

[30] P. Appel, E. Neu, M. Ganzhorn, A. Barfuss, M. Batzer, M. Gratz, A. Tschöpe, and P. Maletinsky, Fabrication of All Diamond Scanning Probes for Nanoscale Magnetometry, Rev. Sci. Instrum. 87, 063703 (2016).

[31] P. Maletinsky, S. Hong, M. S. Grinolds, B. Hausmann, M. D. Lukin, R. L. Walsworth, M. Lonćar, and A. Yacoby, A Robust, Scanning Quantum System for Nanoscale Sensing and Imaging, Nat. Nanotechnol. 7, 320 (2011).

[32] D. Riedel, D. Rohner, M. Ganzhorn, T. Kaldewey, P. Appel, E. Neu, R. J. Warburton, and P. Maletinsky, Low-Loss Broadband Antenna for Efficient Photon Collection from a Coherent Spin in Diamond, Phys. Rev. Applied 2, 064011 (2014).
[33] P. Siyushev, H. Pinto, M. Vörös, A. Gali, F. Jelezko, and J. Wrachtrup, Optically Controlled Switching of the Charge State of a Single Nitrogen-Vacancy Center in Diamond at Cryogenic Temperatures, Phys. Rev. Lett. 110, 167402 (2013).

[34] V. M. Acosta, C. Santori, A. Faraon, Z. Huang, K.-M. C. Fu, A. Stacey, D. A. Simpson, K. Ganesan, S. TomljenovicHanic, A. D. Greentree, S. Prawer, and R. G. Beausoleil, Dynamic Stabilization of the Optical Resonances of Single Nitrogen-Vacancy Centers in Diamond, Phys. Rev. Lett. 108, 206401 (2012).

[35] D. Hunger, C. Deutsch, R. J. Barbour, R. J. Warburton, and J. Reichel, Laser Micro-Fabrication of Concave, LowRoughness Features in Silica, AIP Adv. 2, 012119 (2012).

[36] A. Faraon, P. E. Barclay, C. Santori, K.-M. C. Fu, and R. G. Beausoleil, Resonant Enhancement of the Zero-Phonon Emission from a Color Center in a Diamond Cavity, Nat. Photonics 5, 301 (2011).

[37] E. Janitz, M. Ruf, M. Dimock, A. Bourassa, J. Sankey, and L. Childress, Fabry-Perot Microcavity for Diamond-Based Photonics, Phys. Rev. A 92, 043844 (2015).

[38] I. P. Radko, M. Boll, N. M. Israelsen, N. Raatz, J. Meijer, F. Jelezko, U. L. Andersen, and A. Huck, Determining the Internal Quantum Efficiency of Shallow-Implanted Nitrogen-Vacancy Defects in Bulk Diamond, Opt. Express 24, 27715 (2016).

[39] P. Lodahl, S. Mahmoodian, and S. Stobbe, Interfacing Single Photons and Single Quantum Dots with Photonic Nanostructures, Rev. Mod. Phys. 87, 347 (2015).

[40] D. Najer, M. Renggli, D. Riedel, S. Starosielec, and R. J. Warburton, Fabrication of Mirror Templates in Silica with Micron-Sized Radii of Curvature, Appl. Phys. Lett. 110, 011101 (2017).

[41] S. Bogdanović, S. B. Van Dam, C. Bonato, L. C. Coenen, A. M. J. Zwerver, B. Hensen, M. S. Liddy, T. Fink, A. Reiserer, M. Lonćar, and R. Hanson, Design and LowTemperature Characterization of a Tunable Microcavity for Diamond-Based Quantum Networks, Appl. Phys. Lett. 110, 171103 (2017).

[42] S. B. van Dam, P. C. Humphreys, F. Rozpédek, S. Wehner, and R. Hanson, Multiplexed Entanglement Generation over Quantum Networks Using Multi-Qubit Nodes, arXiv:1702.04885.

[43] N. Kalb, A. A. Reiserer, P. C. Humphreys, J. J. W. Bakermans, S. J. Kamerling, N. H. Nickerson, S. C. Benjamin, D. J. Twitchen, M. Markham, and R. Hanson, Entanglement Distillation between Solid-State Quantum Network Nodes, Science 356, 928 (2017).

[44] K. De Greve, L. Yu, P. L. McMahon, J. S. Pelc, C. M. Natarajan, N. Y. Kim, E. Abe, S. Maier, C. Schneider, M. Kamp et al., Quantum-Dot Spin Photon Entanglement via Frequency Downconversion to Telecom Wavelength, Nature (London) 491, 421 (2012).

[45] I. Aharonovich and E. Neu, Diamond Nanophotonics, Adv. Opt. Mater. 2, 911 (2014).

[46] P. Siyushev, M. H. Metsch, A. Ijaz, J. M. Binder, M. K. Bhaskar, D. D. Sukachev, A. Sipahigil, R. E. Evans, C. T. Nguyen, M. D. Lukin et al., Optical and Microwave Control of Germanium-Vacancy Center Spins in Diamond, arXiv:1612.02947. 
[47] M. Bhaskar, D. Sukachev, A. Sipahigil, R. Evans, M. Burek, C. Nguyen, L. Rogers, P. Siyushev, M. Metsch, H. Park et al., Quantum Nonlinear Optics with a GermaniumVacancy Color Center in a Nanoscale Diamond Waveguide, Phys. Rev. Lett. 118, 223603 (2017).

[48] W. F. Koehl, B. B. Buckley, F. J. Heremans, G. Calusine, and D. D. Awschalom, Room Temperature Coherent Control of Defect Spin Qubits in Silicon Carbide, Nature (London) 479, 84 (2011).
[49] S. Castelletto, B. C. Johnson, V. Ivády, N. Stavrias, T. Umeda, A. Gali, and T. Ohshima, A Silicon Carbide Room-Temperature Single-Photon Source, Nat. Mater. 13, 151 (2013).

[50] D. Riedel, F. Fuchs, H. Kraus, S. Väth, A. Sperlich, V. Dyakonov, A. A. Soltamova, P. G. Baranov, V. A. Ilyin, and G. V. Astakhov, Resonant Addressing and Manipulation of Silicon Vacancy Qubits in Silicon Carbide, Phys. Rev. Lett. 109, 226402 (2012). 